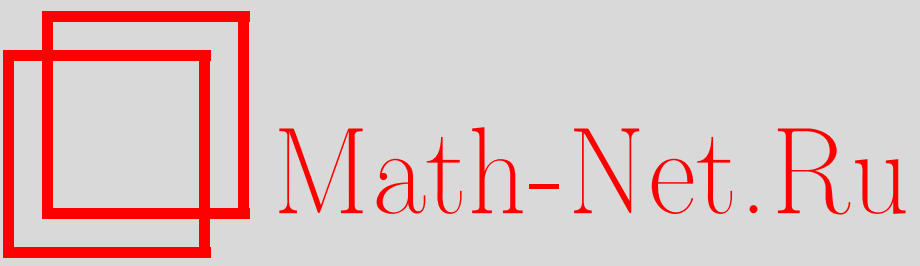

С. П. Новиков, А. С. Шварц, Дискретные лагранжевы системы на графах. Симплекто-топологические свойства, УМН, 1999, том 54, выпуск 1, 257-258

DOI: https://doi.org/10.4213/rm126

Использование Общероссийского математического портала Math-Net.Ru подразумевает, что вы прочитали и согласны с пользовательским соглашением

http://www.mathnet.ru/rus/agreement

Параметры загрузки:

IP : 35.173 .219 .149

26 апреля 2023 г., 11:51:25 


\title{
ДИСКРЕТНЫЕ ЛАГРАНЖЕВЫ СИСТЕМЫ НА ГРАФАХ. СИМПЛЕКТО-ТОПОЛОГИЧЕСКИЕ СВОЙСТВА
}

\author{
С. П. Новиков, А. С. ШвАРц
}

Данная работа развивает [1]-[3], где конструкция Симплектического "Вронскиана" была развита для линейных систем на графах и применялась в теории рассеяния для графов с хвостами.

Рассмотрим одномерный симплициальный комплекс $\Gamma$ (граф), без концов и локально конечньй, т.е. в любой вершине $P \in \Gamma$ сходится лишь конечное число ребер $T_{k}$. Зададим в каждой вершине $P$ гладкое многообразие $N_{P}$ с локальными координатами $x_{P}$. Пусть задано семейство $S$ конечных подмножеств $\alpha$ вершин графа Г. Вершины далее занумерованы индексом $j, P \rightarrow j(P)$.

Пусть задан также набор гладких функций $\Lambda^{\alpha}: N_{j_{1}} \times \cdots \times N_{j_{|\alpha|}} \rightarrow \mathbb{R}$, от всех вершшн подмножества $\alpha \in S$. Рассмотрим функции $\psi(P)=x_{P}$, сопоставляющие вершине $P \in \Gamma$ точку многообразия $N_{P}$; для них определен "Лагранжиан" и "Уравнение Эйлера-Лагранжа":

$$
L\{\psi\}=\sum_{\alpha \in S} \Lambda^{\alpha}\left(x_{j_{1}}, \ldots, x_{j_{|\alpha|}}\right), \quad \frac{\partial L}{\partial x_{P}}=0, \quad P \in \Gamma .
$$

Дискретные лагранжевы системы изучали Обри и Веселов (см. [4]), где $\Gamma=\mathbb{Z}$ (дискретизированная прямая), и все $\Lambda^{\alpha}, N_{P}$ одинаковы: $\Lambda^{\alpha}=\Lambda, N_{P}=N$.

ОПредЕЛЕниЕ 1. Мы назовем лагранжиан (1) локальным, если диаметр всех множеств $\alpha \in S$ конечен и ограничен сверху единой константой $d(\alpha)<M$ в естественной геодезической метрике графа, где длина каждого ребра равна единице.

ЛЕмма 1. Для каждого множества $\alpha \in S$ можно построить односвязный подграф $\Gamma_{\alpha} \subset \Gamma$ (дерево) диаметра не более чем $M$, содержащий все вершины множества $\alpha \in S$.

Доказательство леммы несложно.

ОпРЕДЕлЕниЕ 2. Мы назовем лагранжиан (1) “древесно представленным”, если каждое множество $\alpha$ есть множество вершин дерева $\Gamma_{\alpha}$ диаметра не более чем $M$.

Лемма 2. Каждая локальная лагранжева задача может быть записана как древесно представленная.

Для каждой пары вершин $P, Q \in \alpha$ с индексами $(j, k)$, соответственно, мы обозначим через $l_{j k}^{\alpha}$ единственньй минимальный путь из $P$ в $Q$, лежащий в дереве $\Gamma_{\alpha} \ni l_{j k}^{\alpha}, \partial\left[l_{j k}^{\alpha}\right]=Q-P$.

Определим 2-форому $\Omega$ на бесконечном прямом произведении $N^{\infty}=\prod_{P \in \Gamma} N_{P}$ с локальными координатами $\left(\bigcup_{j} x_{j}\right)$ :

$$
\Omega=\sum_{\substack{\alpha \in S \\ P, Q \in \alpha}} \frac{\partial^{2} \Lambda^{\alpha}}{\partial x_{j} \partial x_{k}}\left[l_{j k}^{\alpha}\right] d x_{j} \wedge d x_{k}=\sum_{\alpha} \Omega^{\alpha},
$$

где $j, k$ - индексы, отвечаюшие вершинам $P, Q \in S,\left[l_{j k}^{\alpha}\right]$ - одномерная цепь в дереве $\Gamma \alpha$, указанная выше.

Мы рассматриваем только локальные древесно представленные вариационные задачи.

Работа выполнена при частичной поддержке Российского фонда фундаментальных исследований (грант № 96-01-00166). 
Теорема. 1) Форма $\Omega$ корректно определена, т.е. каждому ребру $T \subset \Gamma$ отвечает обычная конечномерная 2-форма, зависящая от конечного числа переменных $x_{j}$;

2) Эта форма замкнута: $d \Omega=0$

3) На подмногообразии решений уравнения Эйлера-Лагранэса $\delta L=0$, форма $\Omega$ принимает значения в группе открытых циклов $H_{1}^{\text {open }}(\Gamma ; \mathbb{R})$ графа $\Gamma$, т.е. $\partial \Omega=0$ в группе 1-uепей.

ДокАЗАТЕльСтво пункта 1) очевидно из построения. Для доказательства утверждения 2) следует использовать факт, что каждое $\Gamma_{\alpha}$ - это дерево. Здесь нужно подсчитать вклад в $d \Omega^{\alpha}$ для любого заданного ребра $T \subset \Gamma_{\alpha}$. Заметим, что уравнение Эйлера-Лагранжа на этом этапе не используется. Для доказательства утверждения 3) мы должны рассмотреть $\partial \Omega$, где граница берется в группе 1-цепей.

Зафиксируем вершину $P$ с номером $j$ и рассмотрим границу ( $k$-номера точек $Q \in \alpha)$ :

$$
\left.\partial \Omega\right|_{P}=\sum_{\alpha} \sum_{k} \frac{\partial^{2} \Lambda^{\alpha}}{\partial x_{j} \partial x_{k}} d x_{j} \wedge d x_{k}=\sum_{k} \frac{\partial}{\partial x_{k}}\left(\sum_{\alpha} \frac{\partial \Lambda^{\alpha}}{\partial x_{j}} d x_{j}\right) \wedge d x_{k}
$$

Из уравнений Эйлера-Лагранжа мы имеем:

$$
\sum_{\alpha} \frac{\partial \Lambda^{\alpha}}{\partial x_{j}} d x_{j}=d x_{j}\left(\sum_{\alpha} \Lambda^{\alpha}\right)=0
$$

Теорема доказана.

ЧАСТныЕ СлУчАИ. 1. Пусть $\Gamma=\mathbb{Z}$. В этом случае можно проверить, что формула (2) совпадает с симплектической структурой, введенной Веселовым в [4], хотя это не сразу очевидно. Здесь $H_{1}^{\text {open }}(\mathbb{R} ; \mathbb{R})=\mathbb{R}$ и форма $\Omega$ числовая.

2. Пусть лагранжева задача (1) задается парным взаимодействием, т.е. $\Lambda^{\alpha}$ зависит лишь от пар точек $\Lambda^{\alpha}(P, Q), d(P, Q)<M$. Древесное представление задается выбором минимального пути $l_{P Q}$, соединяющего две взаимодействующие точки, $\Gamma_{\alpha}=l_{P Q}$. В этом случае пункт 2) теоремы очевиден, так как все третьи производные по переменным трех разных точек тождественно равны нулю.

ЗАДАчА. Исследовать 2-форму $\Omega / \delta L=0$, заданную в виде ряда (2), для нелокальньх парных взаимодействий, убывающих с расстоянием, особенно для $\Gamma=\mathbb{Z}^{n}$ или для $\Gamma$ - однородного дерева? Для $\Gamma=\mathbb{Z}^{n}$ сходимость ряда может зависеть от древесного представления, которое неединственно.

\section{СПИСОК ЛИТЕРАТУРЫ}

[1] Новиков С. П. // УМН. 1997. Т. 52. №6. С. 177-178. [2] Novikov S. // Arnoldfest, Additional Volume, Fields Institute, Toronto (to appear in the additional volume dedicated to the 60th birthday of V. Arnold). [3] Novikov S. // Asian J. Math. (to appear in the special volume dedicated to the 70th birthday of Mikio Sato). [4] Веселов А. // УМН. 1991. Т. 46. №6. С. 3-45. 